\title{
De LA TECNOLOGÍA A LA CIENCIA FUNDAMENTAL
}

From technology to the fundamental science

Juvenal Castromonte Salinas ${ }^{1}$

$\checkmark$ academia Real de Ciencias de Suecia, el 2 de octubre pasado, hizo público los nombres de los nuevos ganadores del Premio Nobel de Física "por los revolucionarios inventos en la física de láseres". Los galardonados fueron Arthur Ashkin (96 años), Gérard Mourou (74 años) y Donna Strickland (59 años). La distinción fue conferida en dos mitades, la primera a Ashkin por el invento de las pinzas ópticas y su aplicación para el estudio de sistemas biológicos. La segunda mitad, la dividieron entre Mouruou y Strickland por el desarrollo del método para la generación de impulsos ópticos supercortas de muy alta intensidad. Gracias a este método se ha podido incrementar la potencia de los sistemas de láser, permitiendo desarrollos de aceleradores compactos de partículas, sin perder la capacidad de los aceleradores tradicionales.

El Premio Nobel 2018 fue otorgado por trabajos realizados y publicados hace ya más de 30 años. Los logros de estos tres científicos, desde hace bastante tiempo, forman parte de la ciencia y tecnología clásica; sin embargo, se debe tener presente que cada una de las dos

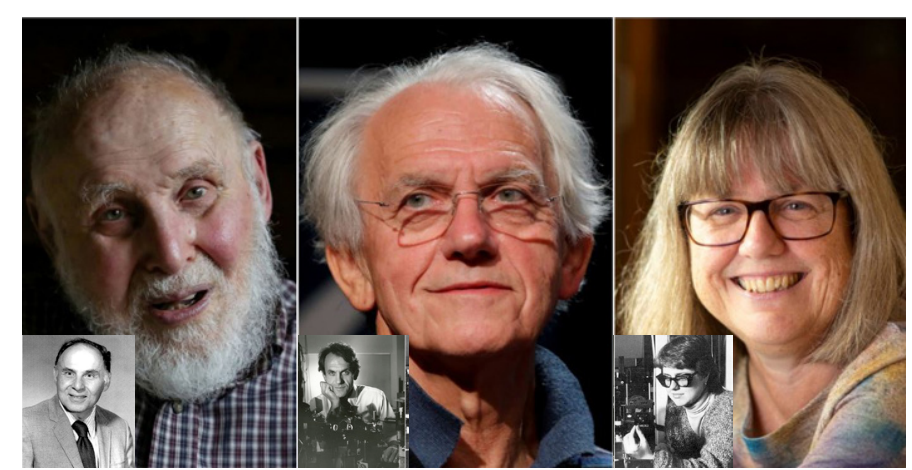

De izQuierda a derecha: Arthur AshKin, Gérard Mourou y Donna STRICKLAND. (LAS Fotos PRINCIPALES HAN Sido tOMADAS DEL PORTAL dE VOA NEWS; LAS

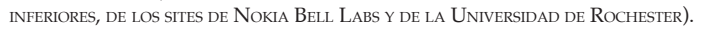

mitades tienen aplicaciones completamente diferentes, que han influenciado grandemente en otras áreas de la ciencia.

La formulación oficial no solo resalta los méritos de los nuevos galardonados sino también evidencia que se trata de investigaciones aplicadas con una orientación tecnológica bastante clara, como sucedió, por última vez en 2014, cuando se premió por el invento de los diodes azules a tres científicos japoneses. Los premios del 2015 al 2017 se confirieron por

1 Profesor Principal. Departamento de Ciencias Exactas, Facultad de Ciencias y Filosofía, Universidad Peruana Cayetano Heredia. 
investigaciones fundamentales (básicas). Otra particularidad es que los premios del 2014 y el 2018 son por trabajos en física óptica que en los últimos años han fortalecido tanto a la física fundamental como a la tecnología. Los inventos de Ashkin, Mourou y Strickland han ampliado significativamente las aplicaciones prácticas de la presión de la luz gracias al progreso en el desarrollo de generadores ópticos cuánticos, concretamente de los láseres que es lo común en el trabajo de los tres científicos.

La hipótesis sobre la presión de la luz no es nueva ya que, en 1619 con la ayuda de esta hipótesis, Kepler pudo explicar el porqué la cola de un cometa se orienta en sentido opuesto a la dirección del Sol. (1) En general, fue un acierto lo de Kepler, solo que debe precisarse que la cola de un cometa se forma bajo la acción de los vientos solares.

En 1873, a partir de sus ecuaciones, Maxwell dedujo la existencia de la presión de la luz y de cualquier tipo de radiación electromagnética. Estas predicciones se comprobaron, entre los años 1899 y 1901, en los trabajos de Lebedev, Ernest Fox y Gordon Ferrie.

Lapresión dela luz visiblees "exageradamente" débil.Para tener una idea de esto, se puedehacer el paralelo de la fuerza con el que la luz solar repele a la Tierra, que es aproximadamente 60 trillones de veces menor que la atracción solar. En 1905, en la Sociedad Británica de Física, Poynting comunicó que los experimentos, para determinar la magnitud de la presión de la luz, habían demostrado que estos efectos son tan insignificantes que "pueden ser excluidos de cualquier fenómeno en condiciones terrestres". Esta conclusión fue válida hasta la aparición de los láseres.

El laser tiene propiedades únicas, como es la de su pureza espectral que ofrece la posibilidad de generar radiación monocromática casi ideal y su altísima coherencia espacial, permitiendo la posibilidad de focalizar en un punto cuyo diámetro no es mayor al de una longitud de onda. Un láser de unos cuantos vattios puede proporcionar una intensidad de radiación que supera en varios miles a la intensidad de la luz visible proporcionada por el Sol. Las posibles aplicaciones que se puedan imaginar son múltiples, uno de ellos es que permite comunicar a las partículas pequeñas una aceleración superior en más de un millón de veces a la aceleración de la caída libre en la Tierra.

Fue precisamente Arthur Ashkin uno de los primeros en valorar estas propiedades únicas de los laseres. A inicios de los años 1960s, Ashkin realizó experimentos con láser en los Laboratorios Bell, como resultado construyó la trampa luminosa con las que se podían sostener objetos pequeñísimos, sin importar su naturaleza. Estas investigaciones le tomaron alrededor de un cuarto de siglo; así, Ashkin y sus colaboradores publicaron el primer artículo, con la descripción de atrapamientos de partículas dieléctricas de dimensiones desde algunas decenas de nanómetros hasta algunas decenas de milímetros, en 1986. Este artículo, de apenas tres páginas, apareció en la revista Optics Letters, donde describió los resultados de investigaciones científicas fundamentales y trascedentes de casi 25 años; esta forma compacta de presentar resultados trascendentes se encuentra en no pocos trabajos de esta envergadura. ${ }^{(2)}$

Con el tiempo, la trampa de luz de Ashkin fue denominada pinza óptica o pinzeta láser (optical tweezers, laser tweezers). En los años siguientes, esta tecnología se desarrolló, perfeccionó y fortaleció grandemente, ampliando de manera significativa sus posibilidades de uso. Las pinzas ópticas, actualmente, no solo 
sostienen o atrapan objetos microscópicos o nanométricos sino también permitieron moverlos de su lugar, hacerlos rotar y cortarlos en partes. Esto ha permitido su amplio uso en la biología molecular, genómica, virología y muchas otras aplicaciones, razón por lo que se cuenta con una de estas pinzas en el LID de la Facultad de Ciencias de la UPCH. Otro importante campo de aplicación de las pinzas ópticas es el enfriamiento por láser de átomos neutros hasta alcanzar temperaturas ultrabajas. Precisamente, por este tipo de trabajo, Chu, Phillips y Cohen-Tannoudji, ex colaboradores de Ashkin ganaron el Premio Nobel de 1997. (4)

Como se mencionó anteriormente, la segunda mitad del premio correspondió a Gérard Mourou y Donna Strickland por sus trabajos en desarrollar métodos efectivos para aumentar la potencia energética de los pulsos de láser. Para entender la importancia de esto es necesario nuevamente retornar al pasado.
La historia de los láseres comerciales empezó en mayo de 1960 cuando Theodore Maiman en Hughes Research Laboratories construyó el primer láser de rubi. Medio año después, en los laboratorios de la corporación IBM, Peter Sorokin y Mirek Stevenson construyeron un láser infrarrojo que solo funcionaba a temperaturas de hidrógeno líquido, que no tenia mayor importancia práctica. En diciembre de 1960, Alí Javan, William Bennett y Donald Herriott, en los laboratorios Bell, construyeron el primer láser de gas que era una mezcla de helio y neón, actualmente muy difundido. Así nació la competencia, que hasta el día de hoy no se detiene, de la creación de nuevos láseres y cada vez mejores.

Reiteramos que un láser de luz focalizado garantiza una alta intensidad de radiación. A inicios de los años 1960s era del orden de $1010 \mathrm{w} / \mathrm{cm}^{2}$, diez años mas tarde se hablaba del orden de $1015 \mathrm{w} / \mathrm{cm}^{2}$. Sin embargo, este
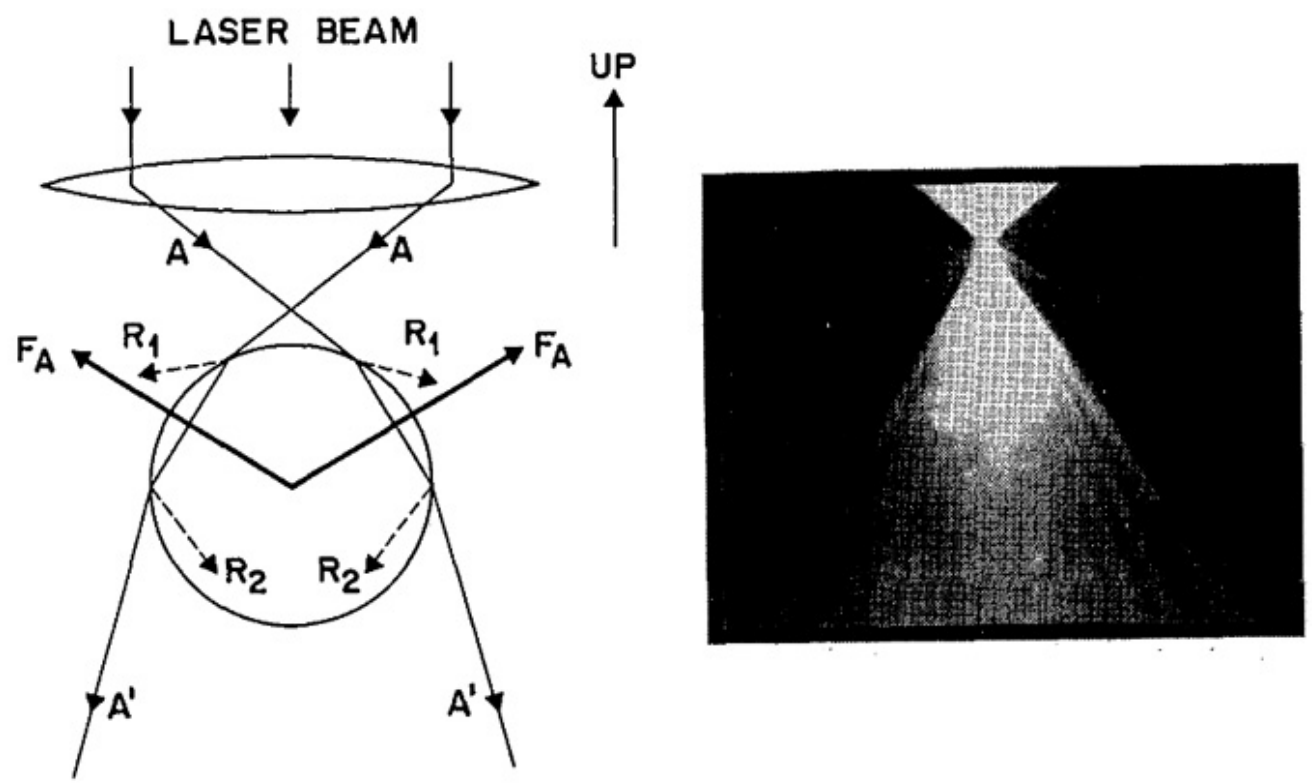

Figura 1 [Tomada de la ref. (2)]. A la izquierda: esquema de atrapamiento de una partícula Mie (pequeños cuerpos esféricos de material dieléctrico, cuyo radio es mayor en varias veces a la longitud de onda, que se encuentra en agua, en una trampa de láser. (3) La fuerza resultante FA dirigida hacia arriba al encuentro de los rayos láser incidentes aparece gracias a la transmisión del impulso de los rayos a la partícula. A la derecha: fotografía del experimento real, donde se sostiene una esfera de $10 \mu \mathrm{m}$ de diámetro. 
ritmo de progreso se frenó hacia mediados de los años 1980s. La situación cambia radicalmente a mediados del año 1985 cuando Mourou y Strickland, que entonces trabajaban en el Laboratory For Laser Energetics de la Universidad de Rochester, publicaron el artículo "Compression of amplified chirped optical pulses", que también es de tres páginas, donde presentaron la descripción del método creado por ellos. (5) La potencia de los pulsos de láser nuevamente empezó a crecer, y que actualmente ya alcanza el orden de $1023 \mathrm{w} /$ $\mathrm{cm}^{2}$.

La esencia del método puede ser descrita en tan solo tres oraciones. Se dejan pasar pulsos ultracortos de láser a través de rejillas difractoras, que los "estiran" en varias órdenes con respecto al tiempo (en sus primeros experimentos Mourou y Strickland utilizaron fibra óptica pero luego se darían cuenta que las rejillas resultaron ser más efectivas). Como resultado, el pico de la energía del campo eléctrico del láser decae tanto que atraviesa a través de un amplificador óptico sin alterar la estructura cristalina del amplificador. El pulso amplificado varias veces se hace pasar por un par de rejillas difractoras más, de manera que son comprimidas a su tamaño original. A la salida se obtiene un pulso muy breve pero de muy alta intensidad (ver Figura 4). Desde los primeros experimentos, este método mostro sus bondades y permitió obtener sistemas de láseres de picosegundos con potencia en terawatios. Lo que sigue, es solo técnica y un poco de imaginación.

Los campos de aplicación de los pulsos de laser ultracortos superpotentes son bastante amplios y en diferentes áreas de la ciencia y la tecnología. Suficiente con mencionar que abarca experimentos que van desde la física fundamental hasta las cirugías oftamológicas de problemas de miopía y astigmatismo.

Las perspectivas, con la potencia alcanzada, para las ciencias fundamentales es grande, las decenas de petawattios alcanzados ofrecen
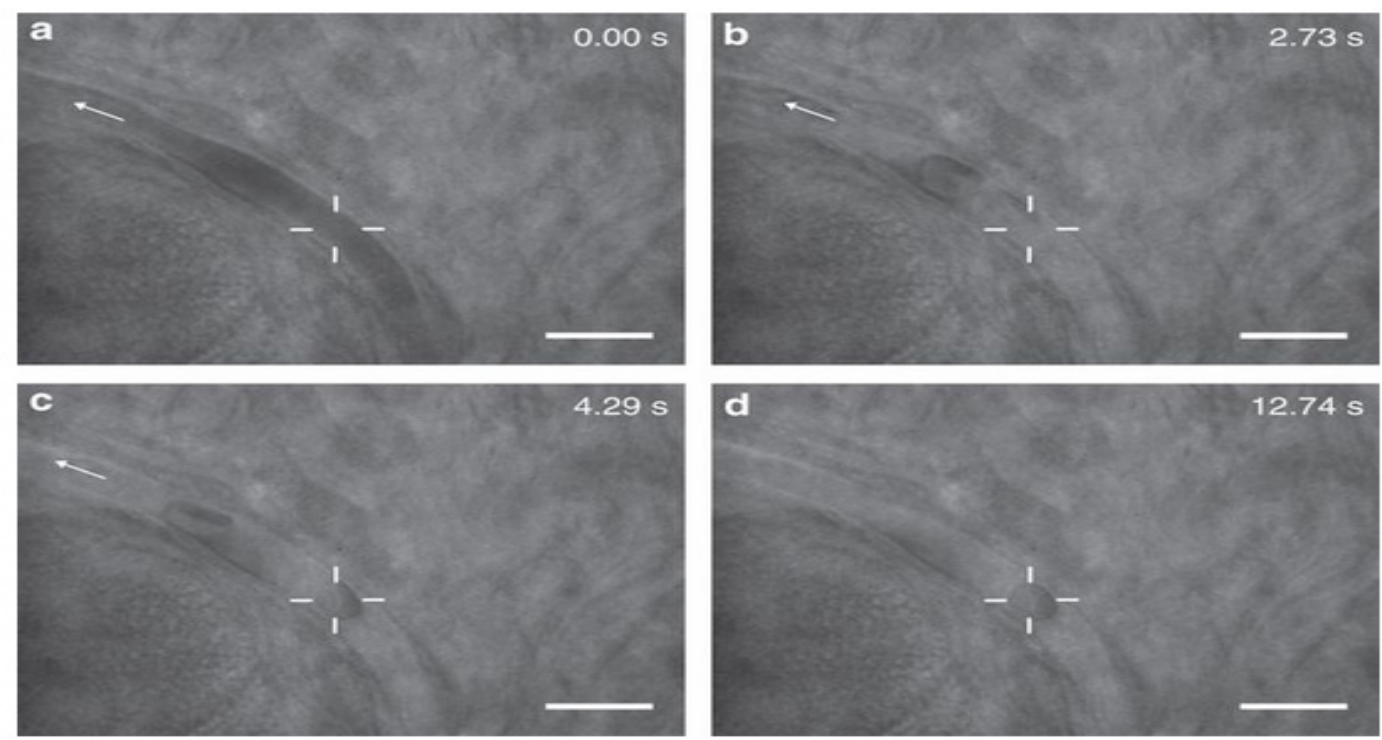

Figura 3. Atrapamiento de un erotrocito en el capilar de un músculo, con la ayuda de la pinza óptica. ${ }^{(6)}$

(a) Al iniciarse el atrapamiento óptico, el flujo sanguíneo es tan rápido que no puede distinguirse una célula. (b) El flujo se enlentece gradualmente tal que puede distinguirse un glóbulo rojo sobre la piza óptica. (c) Después de 4,29 segundos, las pinzas ópticas atrapan un glóbulo rojo mientras que otros siguen pasando. (d) El glóbulo rojo es retenido en la trampa y los otros siguen fluyendo. (Escala de la barra, $10 \mu \mathrm{m}$, "+" indica la trampa óptica y las flechas indican el flujo sanguíneo). 


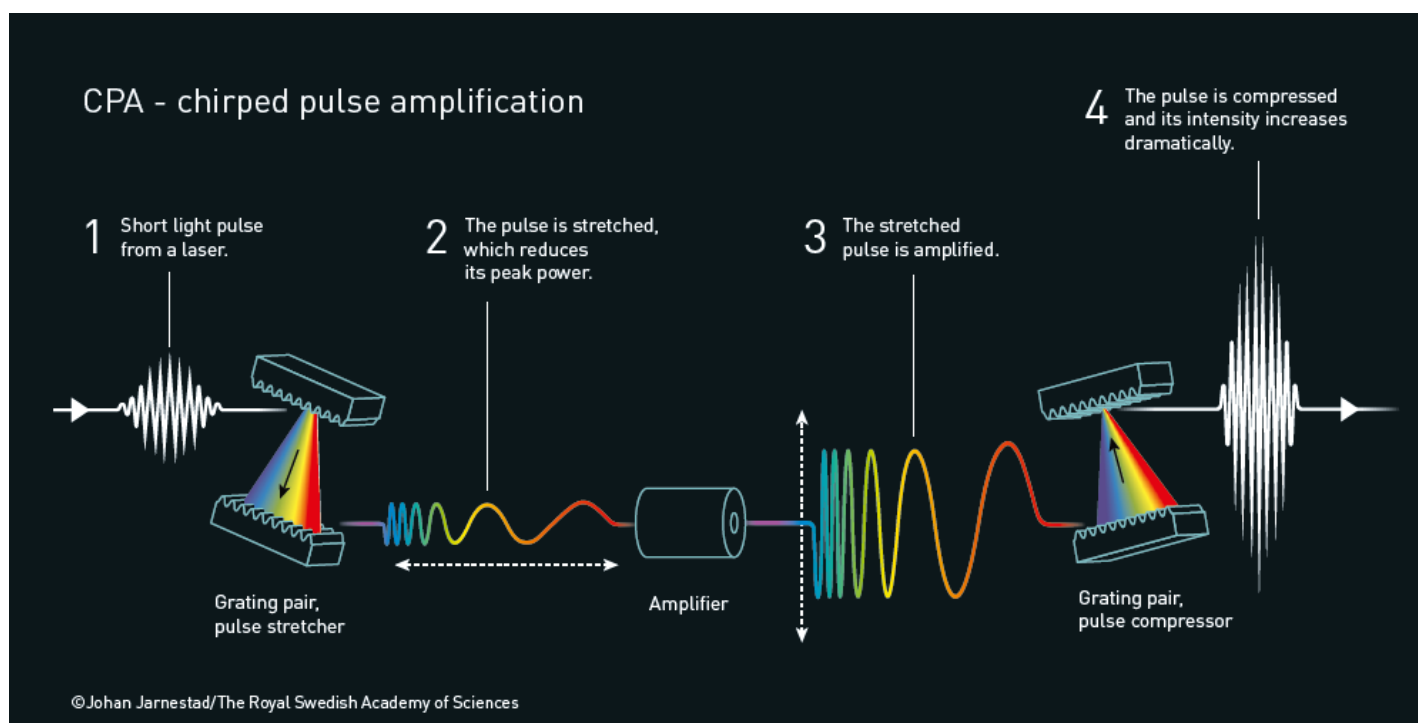

Figura 4. Esquema de trabajo del método CPA. Figura tomada de Nobel Press Release. Ref. (7).

una ventana con posibilidades reales para estudiar las propiedades cuánticas del vacío. La intensidad de la radiación permite generar fotones gamma con altas energías que decaen en el par positrón-electrón.

Finalmente, a manera de curiosidad. Donna Strickland dirige el grupo de láser superrápidos de su universidad. En 1997, ella fue nombrada en la categoría de profesora auxiliar (Assistant Professor) y en todo este tiempo ella apenas ha pasado a ser profesora asociada. Cuando el 2 de octubre del 2019 un corresponsal de la BBC le preguntó porque ella no era profesor principal (Full professor), su respuesta fue simple "I never applied". Este es el tipo de persona la nueva Premio Nobel.

\section{Referencias Bibliográficas}

1. Kepler, Johannes. De cometis libelli tres. URL disponible en: http:/ / doi.org/10.3931/e-rara-1007

2. Ashkin A. et al. Observation of a single-beam gradient force optical trap for dielectric particles. Optics Letters. 1986; 11: 288-290. URL disponible en: https://doi. org/10.1364/OL.11.000288

3. https://en.wikipedia.org/wiki/Mie_scattering

4. https://www.nobelprize.org/prizes/ physics / 1997 / summary $/$

5. Donna Strickland and Gerard Mourou.Compression of amplified chirped optical pulses. Optics Communications. 1985; 56 (3):219-221. URL disponible en: https:// doi. org/10.1016/0030-4018(85)90120-8

6. Zhong $\mathrm{M}$ Ch et al. Trapping red blood cells in living animals using optical tweezers. Nature Communications. 2013; Vol. 4, Article number: 1768

7. https://www.nobelprize.org/uploads/2018/10/pressfig3-fy-en-cpa.pdf

\section{CORRESPONDENCIA:}

juvenal.castromonte@upch.pe 